\title{
An Approach to Identify miRNA Associated with Cancer Altered Pathways
}

\author{
Giovanna Maria Ventola ${ }^{2}$, Antonio Colaprico ${ }^{2}$, Fulvio D'Angelo ${ }^{2}$, \\ Vittorio Colantuoni ${ }^{1}$, Giuseppe Viglietto ${ }^{3}$, \\ Luigi Cerulo $^{1,2}$, and Michele Ceccarelli ${ }^{1,2}$
}

1 Dep. of Science and Technology, University of Sannio, Benevento, Italy

2 BioGeM, Institute of Genetic Research "G. Salvatore", Ariano Irpino, AV, Italy

3 Dep. of Experimental and Clinical Medicine, University of "Magna Graecia", Catanzaro, Italy

\begin{abstract}
MicroRNAs play an important role in the regulation of gene expression by binding mRNA targets causing their degradation or blocking their translation. Several genes has been found to be implicated as miRNA targets in different types of malignant tumors suggesting their involvement in cancer pathogenesis. Detecting direct miRNA-targets associations is not straightforward as in principle targets expressions are not altered except when they are completely repressed by the degradation complex.

In this paper we propose an approach to identify direct miRNAtargets associations hypotheses by means of indirect association measures such as mutual information. Indirect regulons of miRNA and Transcription Factors (TFs) are compared with the Fisher's exact test to identify potential co-regulations which may constitute potential miRNA-TF direct associations.

We apply the method on two cancer datasets, Colon and Lung, drawn from the Cancer Genome Atlas (TGCA) obtaining promising results.
\end{abstract}

Keywords: miRNA, reverse engineering, gene regulatory networks.

\section{Introduction}

MicroRNAs (miRNAs) are small noncoding, endogenous, single-stranded RNA molecules of approximately 22 nucleotides. They play an important role in the regulation of gene expression by binding mRNA targets causing mRNA degradation or blocking mRNA translation. About 5300 human genes are found to be implicated as targets of miRNAs and have function in various human diseases [1. In particular, deregulation of miRNAs in different types of malignant tumors suggests their involvement in the pathogenesis of cancer. Similarly to oncogenes the over-expression of miRNAs may contribute to tumor formation if they bind to tumor suppressor genes, instead, they act as onco-suppressors if they bind oncogenes slowing down cancer progression [15. Such a behavior makes the study of miRNA regulation associations crucial in cancer diagnosis, prognosis and therapy. For instance, Calin et al. found that two genes, coding

A. Petrosino, L. Maddalena, P. Pala (Eds.): ICIAP 2013 Workshops, LNCS 8158, pp. 399-408, 2013.

(C) Springer-Verlag Berlin Heidelberg 2013 
for $m i R-15$ and $m i R-16$, are located within a $30 \mathrm{~kb}$ region of loss in chronic lymphocytic leukemia and that both genes are deleted or down-regulated in the majority of cases [6]. The repression activity modulated by miRNAs depends on the recognition of binding sites located mainly on the 3' untranslated regions of target mRNAs [1]. In order to understand the role of miRNAs in biological processes, it is important to identify their gene targets and which conditions make such binding pivotal. In addition to experimental techniques to identify miRNAtarget interactions, several computational algorithms have been developed to predict putative targets [16]. The most common prediction algorithms identify miRNA targets using sequence-based approaches and rank the candidate targets by considering the sequence complementarity between miRNA seeds and genes 3'-UTRs. Such algorithms yield usually a very high number of false positives. On the other hand, these tools, tested against the available experimentally validated miRNA-target pairs, are not able to predict every known gene target them (false negatives) [3. Alternative approaches exploit reverse engineering algorithms to reconstruct miRNA-gene regulatory networks. For instance, Zhao et al. analyzed the feed-forward loop consisting of miRNAs, transcription factors (TFs) and genes to construct a miRNA-TF regulatory network in glioblastoma and identified miRNA components involved in the Notch signaling pathway [20]. Genovese et al. adopted a network-modeling algorithm to compute pairwise measures of associations and identified some important miRNA, such as miR-34a proposed as a novel regulator of TGF- $\beta$ signaling 8]. The algorithm evaluates the mutual information of miRNA-mRNA pairs. This analysis resulted in the identification of some putative interactions, whose only the minimal proportion $(0.17 \%)$ was predicted to be direct.

In this paper we propose an approach inspired by the work of Genovese et al. to identify potential miRNA-TF associations in cancer altered pathways. In principle the expression of miRNA's targets is not altered except when they are completely repressed by the degradation complex. Thus expression correlation methods, such those adopted by Genovese et al., are not able to identify such direct relationships. To avoid such a limitation we adopt an indirect approach. The regulons of both miRNA and TF are computed through the mutual information of their expression profiles. Such regulons, that comprises both direct and indirect associations, are then compared by means of Fisher's exact test to identify miRNA-TF co-regulations. We show on two cancer datasets that such a computed co-regulation is a valuable criterion to suggest direct miRNA-TF associations. We test our approach on two datasets of common malignant tumors, colon adenocarcinoma (COAD) and lung squamous cell carcinoma (LUSC), obtaining a relevant number of strong literature confirmed interactions, such as $m i R-17 / m i R-20 a \rightarrow B C L 241014$.

The paper is organized as follows. The next Section covers material and methods detailing the proposed approach and defining the evaluation procedure adopted. Section 3 reports and discusses the results obtained by applying the approach on two datasets. Section 4 draws conclusions and outlines future directions. 


\section{Material and Methods}

\subsection{Datasets}

We test our approach on two datasets drawn from The Cancer Genome Atlas (TCGA) portal (http://cancergenome.nih.gov/): Colon Adenocarcinoma (COAD) and Lung Squamous cell Carcinoma (LUSC). The COAD dataset contains the expression levels of 1046 miRNAs in 187 tumor samples obtained with Illumina Genome Analyzer miRNA Sequencing and the expression level of 20531 genes in 193 tumor and 18 normal samples obtained with IlluminaHiSeq RNASeqV2. The LUSC dataset contains the expression levels of 1046 miRNAs in 213 tumor and 45 normal samples obtained with Illumina HiSeq miRNASeq and the expression level of 20531 genes in 259 tumor and 35 normal samples obtained with IlluminaHiSeq RNASeqV2.

\subsection{The Proposed Approach}

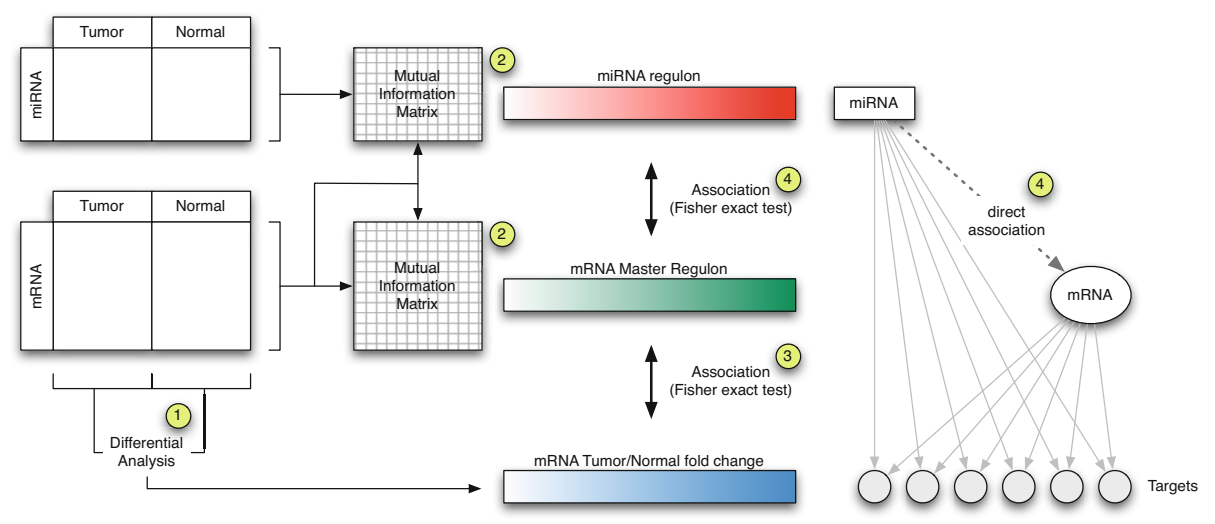

Fig. 1. The proposed analysis workflow

Our aim is to identify hypothesis of association between miRNAs and targets in the context of cancer altered pathways. We adopt the workflow shown in Figure 11 which consists of 5 steps detailed below:

Step1: Differential expression analysis. To determine whether a gene is differential expressed, we apply a test of hypothesis and the fold-change between two conditions, e.g. tumor and normal. In particular we adopt the quantileadjusted conditional maximum likelihood ( $\mathrm{qCML}$ ) method for experiments with single factor, implemented in the edgeR package from Bioconductor [17. Compared against several other estimators, $\mathrm{qCML}$ is the most reliable in terms of bias on a wide range of conditions and specifically performs best in the situation of many small samples with a common dispersion. The p-values generated from the 
analysis sorted in ascending order, are corrected using the Benjamini-Hochberg procedure for multiple testing correction.

Step2: miRNA and TF regulon estimation. Network inference, which is the reconstruction of biological networks from high-throughput data, can provide valuable information about the regulation of gene expressions in cells. Several methods have been proposed in literature [19]. Among all we adopt mutual information implemented in the parmigene $\mathrm{R}$ package, as it is particular efficient for large dataset. The mutual information provides us with an index of dependence between miRNAs and genes and between TFs and genes. We estimate an appropriate threshold with a permutation test to filter out non significant dependences and thus obtaining the set of genes regulated (directly or indirectly) by miRNAs and TFs.

Step3: Transcription factor master regulon estimation. Master Regulators are those transcription factors whose targets are enriched for a particular gene signature (e.g. differentially expressed genes). The enrichment is evaluated using a statistical test, such as Fishers Exact Test. The objective is to place the signature genes within a regulatory context and identify the Master Regulators responsible for coordinating their activity, thus highlighting the regulatory apparatus driving phenotypic differentiation [7. We adopt Fisher's Exact Test to perform an enrichment of TF-regulons obtained in step 2 respect to differentially expressed genes obtained in step 1, identifing the TFs master regulators. The known list of 1503 transcription factors in human is obtained from TRANSFAC 22. The p-values are corrected using the Benjamini-Hochberg $(\mathrm{BH})$ procedure for multiple testing correction.

Step4: miRNA-mRNA association estimation. As for Step 3 we adopt Fisher's Exact Test to perform an enrichment of miRNA-regulons obtained in Step 2 with respect to the master regulons of TFs obtained in Step 3. This analysis let us to identify the most significant direct associations between miRNAs and TFs through the indirect associations with their regulons obtained by means of mutual information.

Step5: post-filtering. To further enrich the set of associations obtained with the previous steps we propose two post-filtering steps:

1. Directional interaction. The association of a miRNA with a TF could determine or not the degradation of the TF mRNA. Consequently the TF expression could be down-regulated, in the case of degradation, or at least unchanged, when mRNA degradation does not occur, allowing mRNA stability and accumulation. To this aim we filter out up-regulated TFs as they are non representative of the canonical functioning of miRNA regulation.

2. Tool prediction. The association of a miRNA with a TF should be supported by the complementarity of miRNA seed sequence with the 3' UTR mRNA. To support such a condition we adopt a representative set of tools provided by miRWalk [5], such as: DIANA-mT, miRanda, miRDB, miRWalk, RNAhybrid, PICTAR4, PICTAR5, PITA, RNA22, and TargetScan. We consider associations predicted by at least 3 tools in at least $5 \mathrm{TF}$. 


\subsection{Evaluation against the Literature}

To evaluate miRNA-mRNA association hypotheses we conduct a survey to test to which extend such hypotheses are confirmed in literature. To this aim we adopt the miRWalk database [5] (http://www.umm.uni-heidelberg.de/ apps/zmf/mirwalk/ ) and IPA $\AA$, (Ingenuity Pathways Analysis, http://www.ingenuity.com). The miRWalk database provides experimentally verified miRNA interaction information obtained from literature. The database, last updated on 15th March 2011, is build semi-automatically through text mining approaches and may provides false positives when for instance the names of miRNAs and TFs co-appears in the article text but are in fact not directly correlated. We assess manually the set of miRWalk validated associations by performing a manual inspection of the referred article and annotate the associations with: text co-occurrence for terms co-occurrence in the published article; expression pattern evidence for less strong validations by high-throughput gene expression experiments, such as microarrays, which not necessarily demonstrate if the miRNA causes the changed expression patterns directly or not; and direct interaction evidence for strong experimental evidence by western blot, qPCR or luciferase reporter assay which accurately validate miRNA target genes. IPA $₫$, is a tool able to identify biological and molecular networks by mapping gene expression data into relevant pathways based on their functional annotation and known molecular interactions. Annotation information comes from published, peer-reviewed scientific publications and is continuously updated. For our purpose we adopt the following IPA enrichment analyses: Biological Functions, that allows for the identification of the biological functions that are most significantly enriched for a given set of genes; Canonical Pathways, that identifies molecular pathways from the IPA library that are significantly associated to a given set of genes; and Network enrichment, that generates regulatory networks of a given set of genes based on their literature known connectivity and constructs networks that optimize for both inter-connectivity and number of focus genes under the constraint of maximal network size.

\section{$3 \quad$ Results and Discussions}

\subsection{COAD}

Starting from the expression values of 20531 genes and applying specific filters, we found 1944 deregulated genes between cancer and normal colon tissues. Among the 1046 miRNAs in COAD dataset, we selected the 105 top expressed miRNAs in cancer condition. The expression data of both genes and miRNAs were then analyzed to calculate their mutual information and estimate the gene regulon for each miRNA. Similarly, we computed the mutual information between the expression profiles of genes and TFs identifying 482 TF master regulators (p-value $<0.01)$, i.e. those that are enriched with differentially expressed genes. By comparing miRNA and master regulons we obtained 61738 significantly associated miRNA-TF pairs $\left(\mathrm{BH}\right.$ corrected p-value $\left.<1.0 \times 10^{-2}\right)$. Those 
Table 1. miRWalk confirmed associations on COAD dataset

\begin{tabular}{l|l|l|l|l|l|l} 
miRNA & TF & FDR & No. of tools & PubMed & Notes \\
\hline \hline hsa-mir-15b & CD36 & $1.0610^{-26}$ & 1 & 17379065 & text co-occurence \\
hsa-mir-181a-1 & CD36 & $8.3510^{-4}$ & 0 & 17379065 & text co-occurence \\
hsa-mir-22 & CD36 & $5.7210^{-25}$ & 0 & 17379065 & text co-occurence \\
hsa-mir-28 & CD36 & $1.9510^{-3}$ & 1 & 17379065 & text co-occurence \\
hsa-let-7c & KIT & $1.5410^{-10}$ & 3 & 18068232 & text co-occurence \\
hsa-mir-17 & KIT & $1.4910^{-24}$ & 3 & 18068232 & text co-occurence \\
hsa-let-7c & MEIS1 & $3.4810^{-70}$ & 1 & 18308931 & text co-occurence \\
hsa-let-7i & MEIS1 & $1.3310^{-9}$ & 0 & 18308931 & text co-occurence \\
hsa-mir-29a & MEIS1 & $8.3510^{-3}$ & 0 & 18308931 & text co-occurence \\
hsa-mir-141 & FOXF1 & $1.0510^{-61}$ & 0 & 18698484 & text co-occurence \\
hsa-mir-200a & FOXF1 & $1.2210^{-103}$ & 0 & 18698484 & text co-occurence \\
hsa-mir-200c & FOXF1 & $1.1610^{-90}$ & 6 & 18698484 & text co-occurence \\
hsa-mir-429 & FOXF1 & $2.8210^{-36}$ & 6 & 18698484 & text co-occurence \\
hsa-mir-20a & BCL2 & 3.21491 & $10^{-7}$ & 5 & 18941111 & direct interaction evidence \\
hsa-mir-17 & MEIS1 & $3.2310^{-187}$ & 0 & 19155294 & text co-occurence \\
hsa-mir-17 & BCL2 & $5.4890810^{-7}$ & 5 & 19666108 & direct interaction evidence \\
hsa-let-7c & MEF2C & $4.2810^{-34}$ & 2 & 19897480 & text co-occurence \\
hsa-mir-143 & KLF4 & $7.8210^{-3}$ & 1 & 20089806 & text co-occurence \\
hsa-mir-27a & MEF2C & $3.6410^{-3}$ & 6 & 20736237 & expression pattern evidence \\
hsa-mir-27b & MEF2C & $3.5410^{-3}$ & 7 & 20736237 & expression pattern evidence \\
hsa-mir-183 & HMOX1 & $7.6810^{-14}$ & 0 & 21147878 & text co-occurence \\
hsa-mir-19b-2 & BCL2 & $5.744710^{-8}$ & 1 & 21883694 & text co-occurence \\
hsa-let-7c & CLU & $3.5710^{-20}$ & 0 & 22749186 & text co-occurence \\
hsa-let-7i & CLU & $6.5310^{-4}$ & 0 & 22749186 & text co-occurence \\
hsa-mir-143 & CLU & $6.0410^{-114}$ & 0 & 22749186 & text co-occurence \\
hsa-mir-200b & FOXF1 & $8.485810^{-117}$ & 6 & 23022474 & text co-occurence \\
\hline
\end{tabular}

represents the potential relationships between miRNAs and TFs that could explain the molecular alterations observed in colon cancer. We examined the 672 most interesting miRNA-TF associations by applying the filters explained in Section 2.2. These associations contains 32 unique miRNAs associated in average with $20 \mathrm{TFs}$. Among the selected miRNA-TF pairs, 27 out of 672 associations corresponded to miRWalk validated miRNA-targets (Table 1).

One of the most interesting result is observed for $m i R-17$ and $m i R$-20a, which share the same group of TFs, thus belonging to the same family. In literature they are widely reported to be up-regulated in colon cancer tissues 41014, promoting cell proliferation, tumour growth and cell cycle progression [14. In our study, $m i R-17$ resulted significantly associated to $31 \mathrm{TFs}, 9$ of which are predicted by at least 3 tools among DIANAmt, miRanda, miRWalk, and TargetScan. One of such associations ( $m i R-17 / m i R-20 a \rightarrow B C L 2)$ has been biologically demonstrated by Beveridge et al. 2]. The fact that such an association is predicted by 5 sequence prediction tools let us suppose for a strong direct target binding association. 
We investigated the enrichment in Biological Functions and Canonical Pathways for $m i R-17$ associated TFs with IPA. Cellular proliferation and differentiation, gene expression and cancer are the most significant biological function categories $\left(\mathrm{BH}\right.$ corrected p-value $<1.0 \times 10^{-6}$ ). Interestingly, in the cancer biological function the first annotations are "metastatic colorectal cancer" and "colorectal tumor", suggesting that the central role of $m i R-17$ in colon tumorigenesis could be mediated by its associated TFs. Moreover, "glucocorticoid receptor signaling" is the only significantly affected canonical pathway suggesting cortisol synthesis in a non-endocrine tumor as recently reported by Sidler et al. [18. To show the putative regulatory network associated with $m i R-17 / m i R$-20a we adopted the IPA network analysis tool that allows for reconstructing the literature known connections among a group of molecules. Figure 2A shows the most significant minimal connected network based on Ingenuity curated annotations and constructed from the set of $31 \mathrm{TFs}$ significantly associated with $m i R-17 / m i R-20 a$. The network reports a total of 35 connected molecules 9 of which are comprised in the initial 31 set and whose principal functions are cell death and survival, cancer, and cell-mediated immune response.

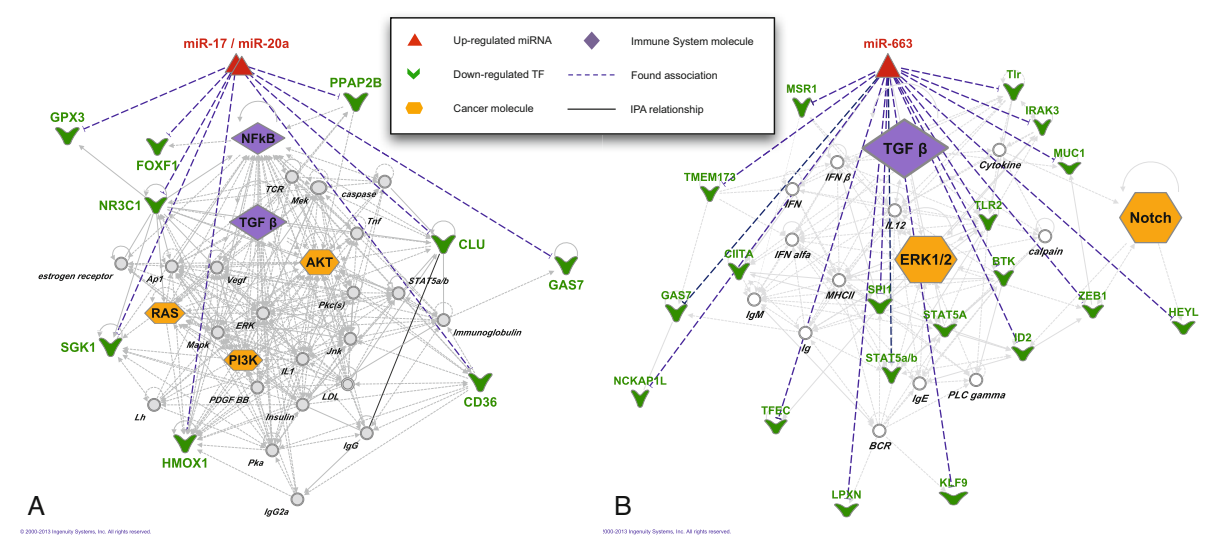

Fig. 2. Enriched biological networks obtained with IPA tool: A) COAD miR-17/miR20a; B) LUSC miR-663

Other interesting associations have been found for miR-200c, miR-221, and miR-429 [92111] but not described due to space limitation.

\section{$3.2 \quad$ LUSC}

The differential expression analysis of LUSC data resulted in 3380 and 274 deregulated genes and miRNAs respectively. Then, we calculated their mutual information to define the gene regulons for miRNAs and we identified $558 \mathrm{TF}$ master regulators $(\mathrm{p}$-value $<0.01)$. miRNA and $\mathrm{TF}$ regulons were compared by Fisher exact test and $11125 \mathrm{miRNA}-\mathrm{TF}$ associations resulted significant (BH corrected 
Table 2. miRWalk confirmed associations on LUSC dataset

\begin{tabular}{l|l|l|l|l|l|l} 
miRNA & TF & FDR & No. of tools & PubMed & Notes \\
\hline \hline hsa-mir-200c & ZEB1 & 2.85 & $10^{-10}$ & 2 & 18376396 & direct interaction evidence \\
hsa-mir-196b & TLR4 & 2.32 & $10^{-4}$ & 0 & 18291670 & text co-occorrence \\
hsa-mir-205 & ZEB1 & 2.12 & $10^{-3}$ & 2 & 18376396 & direct interaction evidence \\
hsa-mir-429 & ZEB1 & 5.28 & $10^{-28}$ & 0 & 18381893 & direct interaction evidence \\
hsa-mir-141 & ZEB1 & 2.62 & $10^{-21}$ & 0 & 18483486 & direct interaction evidence \\
hsa-mir-429 & TBX2 & $3.3910^{-7}$ & 6 & 18698484 & text co-occorence \\
hsa-mir-141 & TBX2 & 1.37 & $10^{-4}$ & 3 & 18698484 & text co-occorence \\
hsa-mir-205 & TBX2 & 2.42 & $10^{-6}$ & 6 & 18698484 & text co-occorrence \\
hsa-mir-96 & ZEB1 & 7.47 & $10^{-13}$ & 0 & 19167416 & text co-occorence \\
hsa-mir-9-1 & TLR4 & 1.71 & $10^{-4}$ & 5 & 19289835 & text co-occorrence \\
hsa-mir-9-1 & TLR2 & $1.5210^{-3}$ & 2 & 19289835 & text co-occorrence \\
hsa-mir-9-2 & TLR4 & $1.0910^{-4}$ & 1 & 19289835 & text co-occorrence \\
hsa-mir-9-2 & TLR2 & 1.15 & $10^{-3}$ & 6 & 19289835 & text co-occorrence \\
hsa-mir-142 & TLR4 & $8.3910^{-7}$ & 2 & 21037424 & text co-occorrence \\
hsa-mir-369 & ZEB1 & $1.6210^{-4}$ & 1 & 21125666 & text co-occorrence \\
hsa-mir-429 & WNT2 & $1.3010^{-10}$ & 2 & 21130073 & text co-occorence \\
hsa-mir-200a & ZEB1 & $7.9510^{-10}$ & 2 & 18376396 & direct interaction evidence \\
hsa-mir-200b & ZEB1 & $5.0210^{-5}$ & 6 & 22261924 & direct interaction evidence \\
hsa-mir-7-1 & TLR4 & 2.95 & $10^{-4}$ & 0 & 23135998 & text co-occorrence \\
\hline
\end{tabular}

p-value $<1.0 \times 10^{-2}$ ). The identified miRNA-TF associations were further analyzed applying the filters described in Section 2.2. We focused on 1136 associations composed by 38 miRNAs that were associated in average with 30 TFs. Comparing the results with literature, 17 out of 1136 miRNA-TF associations were found already described in several studies (Table 21).

Very interesting miRNA-TF associations, for elucidating the role of miRNAs in lung tumorigenesis, were found for $m i R-663$. The overexpression of $m i R-663$ in lung cancer was already reported in a recent study where the authors demonstrated it regulates cancer cell proliferation and apoptosis through targeting TGF $\beta$ [13. In our study, we confirmed miR-663 is highly up-regulated in LUSC, and it resulted significantly associated to $34 \mathrm{TFs}, 9$ of which are predicted by at least 3 tools (such as miRanda, miRWalk, TargetScan, PICTAR5). IPA analysis was performed to evaluate the enrichment in Biological Functions for miR-663 associated TFs. The most significant Biological Function (BH corrected p-value $\left.=6.0 \times 10^{-9}\right)$ is differentiation of cells which was predicted significantly decreased $(\mathrm{z}$-score $=-2.844)$ by IPA algorithm. This biological annotation represents also the top function of a proposed regulatory network built through IPA network analysis tool (Figure2B). Starting from miR-663 associated TFs, this regulatory network shows the known relationships between 35 connected molecules, 17 of which are included in the initial 34 set.

Other remarkable result is observed for $m i R$-141 and $m i R$-200c, which share $37 / 44$ associated TFs and, as expected, belong to the same miRNA family. Interestingly, they were recently reported as novel serum biomarkers associated to 
poor prognosis in LUSC [12. Their associated TFs resulted enriched for cellular differentiation, cell death and immune cell trafficking (BH corrected p-value $<1.0 \times 10^{-6}$ ) through IPA Biological Functions analysis.

\section{Conclusions and Future Work}

We proposed a method to identify potential associations between miRNAs and Transcription Factors playing a central role in altered cancer pathways. The algorithm is able to identify potential direct relationships where other methods, based on pure correlation measures, may fail. It is still affected by false positives thus heuristics able to filter out not relevant associations are necessary. We proposed two filtering heuristics, one based on interaction directionality and another that exploits sequence bases prediction tools.

We test the method on two cancer datasets, Colon Adenocarcinoma (COAD) and Lung Squamous cell Carcinoma (LUSC), showing important literature confirmed associations. Result obtained with this study consolidate the potential involvement of miRNAs in colon and lung cancerogenesis but further studies are necessary to examine all suggested interactions and to elucidate their role in cancer.

Acknowledgements. This work was supported by a research project funded by MiUR (Ministero dell'Università e della Ricerca) under grant FIRB2012RBFR12QW4I.

\section{References}

1. Bartel, D.P.: MicroRNAs: genomics, biogenesis, mechanism, and function. Cell 116, 281-297 (2004)

2. Beveridge, N., Tooney, P., Carroll, A., Tran, N., Cairns, M.: Down-regulation of mir-17 family expression in response to retinoic acid induced neuronal differentiation. Cell Signal 21(12), 1837-1845 (2009)

3. Coronello, C., Benos, P.: Comir: combinatorial microRNA target prediction tool. Nucleid Acid Research (2013)

4. Diosdado, B., van de Wiel, M.A., Terhaar Sive Droste, J.S., Mongera, S., Postma, C., Meijerink, W.J., Carvalho, B., Meijer, G.A.: MiR-17-92 cluster is associated with 13q gain and c-myc expression during colorectal adenoma to adenocarcinoma progression. British Journal of Cancer 101(4), 707-714 (2009)

5. Dweep, H., Sticht, C., Pandey, P., Gretz, N.: miRWalk-database: prediction of possible miRNA binding sites by "walking" the genes of three genomes. Journal of Biomedical Informatics 44(5), 839-847 (2011)

6. Calin, G.A., et al.: Frequent deletions and down-regulation of micro- RNA genes miR15 and miR16 at 13q14 in chronic lymphocytic leukemia. Proceedings of the National Academy of Sciences of the United States of America 99(24), 15524-15529 (2002)

7. Lefebvre, C., et al.: A human B-cell interactome identifies MYB and FOXM1 as master regulators of proliferation in germinal centers. Molecular Systems Biology 6 (June 2010) 
8. Genovese, G., et al.: MicroRNA regulatory network inference identifies miR-34a as a novel regulator of TGF- $\beta$ signaling in glioblastoma. Cancer Discovery 13 (2012)

9. Hur, K., Toiyama, Y., Takahashi, M., Balaguer, F., Nagasaka, T., Koike, J., Hemmi, H., Koi, M., Boland, C., Goel, A.: Microrna-200c modulates epithelial-tomesenchymal transition (emt) in human colorectal cancer metastasis. Gut (2012)

10. Earle, J.S., Luthra, R., Romans, A., Abraham, R., Ensor, J., Yao, H., Hamilton, S.R.: Association of microrna expression with microsatellite instability status in colorectal adenocarcinoma. J. Mol. Diagn. 12(4) (2010)

11. Li, J., Du, L., Yang, Y., Wang, C., Liu, H., Wang, L., Zhang, X., Li, W., Zheng, G., Dong, Z.: Mir-429 is an independent prognostic factor in colorectal cancer and exerts its anti-apoptotic function by targeting sox2. Cancer Lett. 329(1), 84-90 (2013)

12. Liu, X., Zhu, W., Huang, Y., Ma, L., Zhou, S., Wang, Y., Zeng, F., Zhou, J., Zhang, Y.: High expression of serum mir-21 and tumor mir-200c associated with poor prognosis in patients with lung cancer. Med. Oncol. (2011)

13. Liu, Z.Y., Zhang, G.L., Wang, M.M., Xiong, Y.N., Cui, H.Q.: Microrna-663 targets tgfb1 and regulates lung cancer proliferation. Asian Pac. J. Cancer Prev. 12(11), 2819-2823 (2011)

14. Luo, H., Zou, J., Dong, Z., Zeng, Q., Wu, D., Liu, L.: Up-regulated mir-17 promotes cell proliferation, tumour growth and cell cycle progression by targeting the rnd3 tumour suppressor gene in colorectal carcinoma. Biochem. J. 442(2), 311-321 (2012)

15. Nohata, N., Hanazawa, T., Kinoshita, T., Okamoto, Y., Seki, N.: MicroRNAs function as tumor suppressors or oncogenes: Aberrant expression of microRNAs in head and neck squamous cell carcinoma. Auris Nasus Larynx (2012)

16. Reyes-Herrera, P.H., Ficarra, E.: One Decade of Development and Evolution of MicroRNA Target Prediction Algorithms. Genomics, Proteomics \& Bioinformatics 10(5), 254-263 (2012)

17. Robinson, M.D., McCarthy, D.J., Smyth, G.K.: edgeR: a Bioconductor package for differential expression analysis of digital gene expression data. Bioinformatics 26(1), 139-140 (2010)

18. Sidler, D., Renzulli, P., Schnoz, C., Berger, B., Schneider-Jakob, S., Flück, C., Inderbitzin, D., Corazza, N., Candinas, D., Brunner, T.: Colon cancer cells produce immunoregulatory glucocorticoids. Oncoimmunology 1(4), 529-530 (2012)

19. Smet, R.D., Marchal, K.: Advantages and limitations of current network inference methods. Nature Reviews Microbiology 8(10), 717 (2010)

20. Sun, J., Gong, X., Purow, B., Zhao, Z.: Uncovering microRNA and transcription factor mediated regulatory networks in glioblastoma. PLoS Computational Biology (2012)

21. Sun, K., Wang, W., Zeng, J., Wu, C., Lei, S., Li, G.: Microrna-221 inhibits cdkn1c/p57 expression in human colorectal carcinoma. Acta Pharmacol Sin. 32(3), 375-384 (2011)

22. Wingender, E., Dietze, P., Karas, H., Knüppel, R.: TRANSFAC: A Database on Transcription Factors and Their DNA Binding Sites. Nucleic Acids Research 24(1), 238-241 (1996) 